\title{
KEPES, desde Colombia al mundo. Una tarea pendiente: análisis bibliométrico y relacional
}

\begin{abstract}
Resumen
Han pasado 16 años desde el nacimiento de la revista KEPES, por lo que ha sido necesario detenerse en el camino para hacer un recuento histórico de lo que la revista ha desarrollado. El objetivo del presente estudio se basa en la documentación y análisis cuantitavo del contenido y la historia de la revista KEPES. Se ha creado una base de datos con 269 documentos, compuesto por los 20 volúmenes de la revista (2004-2019), a los cuales se les ha realizado diversos estudios cienciométricos. Utilizando indicadores bibliométricos y de relación (Lawani, Subramanyam y el coeficiente de colaboración), los resultados indican que la revista KEPES se ha comportado como una revista masculina, nacional, principalmente de autorías individuales, primando históricamente el ensayo como tipo de escrito para la divulgación del conocimiento en idioma español; con redes de colaboración locales y acotadas. Si bien sus índices de colaboración han aumentado progresivamente, al igual que la estandarización del formato artículo científico, la idea de vincular Colombia con el mundo aún sigue siendo una tarea pendiente. Este análisis permite presentar la historia y tendencia de la revista de autores y relaciones.
\end{abstract}

\author{
Yerco Uribe-Bahamonde \\ Doctor (c) en Ciencias Humanas \\ Universidad de Talca. Talca, Chile \\ Magíster en Psicología Social, \\ Magíster en Diseño de productos \\ Correo electrónico: yuribe@utalca.cl \\ (- orcid.org/0000-0002-1791-404X \\ Google Scholar
}

Recibido: agosto 06 de 2019 Aprobado: junio 15 de 2020

Palabras clave:

Bibliometría, diseño, relación de autores. 


\title{
KEPES, from Colombia to the world. A pending task: Bibliometric and relational analysis
}

\begin{abstract}
Sixteen years have passed since the birth of the journal KEPES, so it has been necessary to stop along the way to make a historical account of what the journal has developed. The objective of this study is based on the documentation and quantitative analysis of the content and history of the journal KEPES. A database with 269 documents has been created, consisting of the 20 volumes of the journal (2004-2019) to which several scientometric studies have been carried out. Using bibliometric and relational indicators (Lawani, Subramanyam and the collaboration coefficient) the results indicate that the journal KEPES has behaved as a male, national, mainly of individual authorship journal, historically prioritizing the essay as a type of writing for the dissemination of knowledge in the Spanish language with local and limited collaboration networks. Although its collaboration rates have progressively increased, as has the standardization of the scientific article format, the idea of linking Colombia to the world is still a pending task. This analysis allows presenting the history and trend of the journal, of the authors and of the relationships.
\end{abstract}

Key words:

list of authors, bibliometrics, design. 


\section{Introducción}

El artículo científico es hoy uno de los principales mecanismos documentadores y divulgadores de nuestro conocimiento científico (Santos, 2010). Muchos cambios técnico-tecnológicos han pasado desde el primer artículo científico instaurado por Henry Oldenburg en el año $1665^{1}$.

Claramente, en 1665 no existían los medios digitales de los que disponemos hoy para la divulgación de conocimientos y la comunicación se entablaba a través de libros, folletos autoeditados o cartas. Lo cual, si se analiza, no está tan alejado de la realidad latinoamericana actual.

Los grupos e instituciones ligadas a la ciencia buscan cada día validarse y compartir sus conocimientos de manera directa a la comunidad, estableciendo espacios críticos y validos de discusión, generando registros de construcción de conocimiento que ayudarán a los siguientes investigadores a avanzar en el saber. Hecho que ha sido rescatado incluso por Isaac Newton "Si he visto más, es pararme sobre los hombros de gigantes" ${ }^{\prime 2}$.

Publicar permite conocer las distintas miradas en campos determinados de conocimiento. Posibilita estar tanto de acuerdo como en desacuerdo con los artículos, citarlos, criticarlo e incluso, algo más importante que lo anterior: inspirar nuevas ideas a partir de las mismas investigaciones, generando a su vez, nuevas publicaciones (Polanco-Carrasco, 2015).

Las nuevas tecnologías de información han ido modificando los principales medios de difusión científica. Internet en este caso, se convirtió en una forma

\footnotetext{
'En marzo de 1665, Henry Oldenburg escribe y difunde su primer número de la revista científica "Philosophical Transactions" para compartir noticias académicas.

2El buscador "Google académico" presenta hasta la actualidad (2019) en su bajada la leyenda: "A hombros de gigantes", en alusión a la famosa cita de Isaac Newton.
} 
de mostrar contenidos de manera más barata, a diferencia del medio impreso (Björk et al., 2014; Wren, 2005).

Internet ha proporcionado nuevos valores a los contenidos: la inmediatez, libertad de almacenamiento, facilidad en el procesamiento, efectividad en la búsqueda, resultando en la inmediatez y exactitud en el análisis de datos (Paños Álvarez, 1999; Rodríguez Piña, 2006).

Uno de los beneficios del almacenamiento y el procesamiento de la información en la ciencia ha recaído en la cienciometría y los análisis bibliométricos. De facto, varios estudios han dado cuenta de la importancia de los análisis bibliométricos para la ayuda del devenir y proyección de las mismas revistas científicas (Pinski \& Narin, 1976; Braun, Glänzel, \& Schubert, 1988; Moed, 2009; Ray Reutzel \& Mohr, 2015), con la finalidad de identificar cuantitativamente los vínculos de colaboración, por medio de las características generales de los autores (sexo, instituciones, edades, jerarquías académicas, etc.); así como el análisis de citas y su proyección en el tiempo, entre otros aspectos (Ray Reutzel \& Mohr, 2015).

La bibliometría es definida como un estudio empírico-cuantitativo, cuyo principal eje es estudiar y analizar la actividad investigativa mediante las publicaciones en revistas científicas (Ghadimi et al., 2019). Puede proporcionar información temporal y proyectiva de los temas de investigación, además de detectar una serie de factores que puedan afectar a una disciplina científica en particular (Fergnani, 2019; Lawani, 1981; Pritchard, 1969; Stelzer et al., 2015).

En América Latina, los análisis bibliométricos han ganado popularidad en los últimos años en diversas revistas, tales como: análisis de la productividad y calidad de artículos clínicos en cirugía (Aravena T, Cartes-Velásquez, \& Manterola D, 2013), los 100 artículos científicos de psicología más importantes 
en Colombia (Cudina \& Ossa, 2016), análisis de la maduración científica (Keathley-Herring et al., 2016), narrativas digitales como nueva estrategia educativa (Villa Enciso et al., 2016). En esta revista en cuestión, KEPES: Identificación de la productividad e influencia de autores iberoamericanos sobre alfabetización informacional (Uribe-Tirado \& Alhuay-Quispe, 2017), estudio mexicano que analiza los primero pasos de la Revista Mexicana de Ciencias Políticas y Sociales a 60 años de su fundación (Serrano, 2016), análisis del impacto de las publicaciones científicas mexicanas relacionadas a los esteroides (Hernandez-Garcia et al., 2016), historia bibliométrica de las revistas científicas en psicología (Tur-Porcar et al., 2018), análisis de 50 años de revistas investigación en transportes (Modak et al., 2019), el análisis de la revista Group Decision and Negotiation a sus 25 años de trayectoria (Laengle et al., 2018), de la misma forma la revista Psykhe al cumplir sus 25 años (Salas et al., 2017).

El presente artículo exhibe un análisis bibliométrico y relacional de $K E P E S^{3}$ Revista del Grupo de estudio en Diseño Visual, editada y dirigida por el Departamento de Diseño Visual de la Universidad de Caldas.

KEPES se encuentra indexada en las siguientes bases de datos: Publindex, LATINDEX, SCOPUS, SRG-INDEX, EBSCO-HOST y MIAR. En este momento, se halla situada en el más alto cuartil (Q1) en la categoría Visual Arts and Performing Arts, presentando un $\mathrm{H}$ index equivalente a 3, según consta en la base de datos de Scimago (Scimago Journal \& Country Rank, 2019). Lo anterior es reflejo de la importancia de factores tales de calidad, impacto e indización característicos de las publicaciones alto impacto o de corriente principal (Valderrama, 2012). La revista se ha editado de manera continua, sin interrupciones y publicándose un volumen semestral hasta el día de hoy, 
publicándose con una frecuencia anual desde el primer número (Ilamado cero "0") hasta el n¹1.

Finalmente, debemos destacar que la revista en su historia no ha tenido un análisis bibliométrico relacional abiertamente discutido. El hecho de cumplir 16 años de edición y su ingreso al podio Q1 en SCOPUS en 20174, genera importantes oportunidades para responder las siguientes preguntas: ¿Cuál ha sido el recorrido histórico de KEPES?, ¿Cuál es la diferencia de participación entre autores? ¿Cuánto se ha colaborado entre las instituciones participantes?

Todo esto, con el fin de poder ayudar a mantenerla y seguir consolidándola aún más en el medio académico, que es altamente competitivo.

\section{Historia de la revista KEPES}

Durante los últimos años hemos asistido a transformaciones considerables en el diseño a nivel global y como tal, el Departamento de Diseño Visual de la Universidad de Caldas ha sido testigo y partícipe de estos cambios estructurales en la imagen y el diseño. (Grupo de estudio en Diseño Visual, 2004)

Así prosan las primeras líneas editoriales del volumen 0 de la revista KEPES, al momento de elaborarlo. Un joven "grupo de estudios el Departamento de Diseño Visual de la Universidad de Caldas" presentaba una idea novedosa en el campo del diseño en América Latina; el interés de instaurar espacios catalizadores de una verdadera reflexión sobre el diseño, culminando con "Dejar la huella sobre el devenir de la investigación en diseño, y todo lo que ello pueda significar" (Ospina, 2010). Para ello, la piedra angular referencial estaba centrada en la obra realizada por el húngaro Georgy Kepes (1906-2001), destacado investigador del diseño, la imagen y la percepción. "Somos conscientes de la responsabilidad de llevar el nombre «KEPES» para identificar nuestro grupo de estudio" (Grupo de estudio en Diseno Visual, 2004). 
KEPES desde su nacimiento ha puesto en manifiesto el valor de la multi y la trans-disciplinariedad en el mundo del estudio del diseño. Y el hecho de estar fundacionalmente compuesto por diseñadores, artistas plásticos, ingenieros, arquitectos y filósofos (Grupo de estudio en Diseño Visual, 2004), le han dado una flexibilidad y una visión cuasi holística a la disciplina.

En 2005, Felipe Londoño vuelve a remarcar y reconocer la dirección científica, fáctica y empírica de la revista a través de sus publicaciones: "Hechos visibles de las metodologías del diseño" (Londoño, 2005). Evento que se repite hasta el número 4; Ospina abre diciendo: "Bien Complejo resulta mantener una publicación que cumpla con los niveles exigidos para divulgación científica" (Ospina, 2008).

En dirección de Ospina se plasma la frustración de no poder llevar la tecnología — propio del diseño_ - al lenguaje de la ciencia y por lo cual, como medida, KEPES deja de lado lo local y se internacionaliza, con lo que se agregan importantes autores de Argentina, España y Venezuela. KEPES comienza a abrirse al mundo.

Los años pasan y KEPES crece. Con las diversas indexaciones y cumplimiento de criterios, la revista está cada vez más cerca de la anhelada validación externa: "Desde su lanzamiento en el año 2004, el comité editorial y demás colaboradores, han trabajado para que la publicación cumpla con todos parámetros que se exigen a los medios de divulgación científica" (Villa, 2011).

En 2014, la revista se empodera en el medio; y fruto de ello es la capacidad de entregar un proceso evaluativo exigente y detallado (Villa, 2014). El medio es competitivo y la capacidad de revisión de todos los artículos enviados se hace dantesco. No obstante, el apoyo tanto del grupo de evaluadores, así como el compromiso de la propia Universidad de Caldas y la Oficina de Revistas 
Indexadas, abre la oportunidad a duplicar periodicidad de publicación de la revista (Villa, 2015a). Este cambio no da más que buenas noticias a KEPES:

"El cambio en la periodicidad de la revista ha tenido un impacto positivo en todos los aspectos de la edición, tanto en el aumento de postulaciones recibidas, como en el número de artículos divulgados anualmente y en la integración a este proceso de nuevos profesores e investigadores adscritos a universidades nacionales e internacionales" (Villa, 2015b).

KEPES en su historia ha sido dirigida por tres directores. En el número 0 "cero" la dirección está velada por el Grupo de Estudio en Diseño Visual, lo que explica la falta de un firmante.

Los dos números siguientes, 1 y 2, es el doctor y arquitecto Felipe César Londoño L. quien dirige la revista durante 2005 y 2006. Desde el número 3 y hasta el 6, el Licenciado en Filosofía y Letras William Ospina Toro asume como director (2007-2010). De ahí en tanto, es el Máster en Estética Gustavo A. Villa Carmona es quién asume la dirección de KEPES hasta el primer semestre de 2019, sucedido en la actualidad por el Dr. Walter Castañeda Marulanda (2020).

\section{Formato y línea gráfica de la revista KEPES a lo largo de su historia.}

A nivel gráfico, KEPES (número cero "0") nace con un formato de carácter experimental y dinámico ${ }^{5}$, el cual varía tanto en diagramación como en tipos formales de fuentes (Andale Mono, Courier, Helvetica, Monaco, Palatino, Skia, Times y Verdana). Estos formatos dinámicos se plantean como una representación ideológica de cada autor y servían de apoyo comunicacional a cada uno de los temas tratados en cada artículo. 
Al diagramar el número 1 de la revista (ver figura 1 -a), esta presentaba un formato vertical de $164 \times 235 \mathrm{~mm}$ (C5). Sumado a que la revista se alinea con los requerimientos de las diversas indizaciones, por lo que define ajustes a su línea gráfica. Al tener como requerimiento los diversos elementos ortotipográficos propios de una revista científica (nombre autor, correos, afiliación, palabras claves, año, volumen, numero, páginas, ISSN, fechas de aprobación y aceptación), se optó por complementar la caja central con una caja anexa, la cual contendría dicha información requerida. Con el fin de presentar toda la información en la primera hoja (tiro) y dejar el anexo de resumen en inglés en el retiro. Más tarde, en el número 2 (ver figura 1-b), la estructura se trabajó en un formato de cuadrado perfecto $(200$ × 200 mm enfrentados, dándole la cualidad de una revista 200 × 400 extendida), haciendo sintonía la estructura cuadrada de la letra " $\mathrm{K}$ " de KEPES.

En cuanto a los párrafos, estos se presentan en un texto justificado a la derecha con bandera a la izquierda, diagramado en una caja cuadrada de texto con ajustes de desplazamiento externo (cuadrado óptico). Todo esto, siempre apoyado del blanco circundante como respuesta al lenguaje de la visión. La fuente utilizada en el cuerpo es "Optima Font", elegida por su legibilidad y lecturabilidad tanto en aplicación impresa como digital. No obstante, esta fuente no es la única, ya que para aspectos de mejora en la lectura, se optó por la diferencia de fuentes entre los títulos, bajadas y cuerpos, entregándole un apoyo al lector en términos de ubicación espacial y jerarquías.

A nivel de diferencia de volúmenes, la revista hasta antes del año 5, no presenta mayor diferenciación que no fuese la declarada en los lomos mediante leyendas. Es por ello por lo que, por motivos de resalte en las exposiciones de bibliotecas, se le agrega el color como elemento diferenciador, integrando este aspecto (color) tanto en el triángulo del logo, como en una ancha franja que transita por la portada, contratapa y lomo. Este elemento diferenciador 
de volúmenes dejó en evidencia una identidad única y contundente a cada compilado de documentos.

El punto del color de cada volumen no es presentado de manera azarosa, sino que se dispone de un abanico de colores definidos desde un inicio por Claudia Jurado por medio de colores, primarios, segundarios y terciarios (imagen 1).
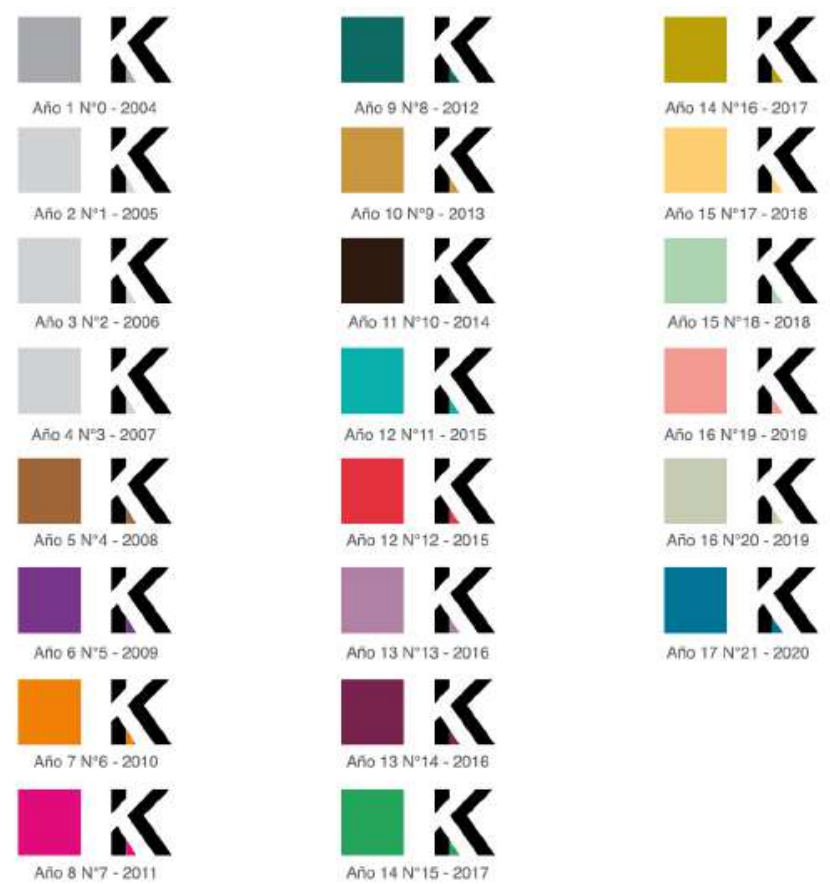

Imagen 1. Gama de colores histórico de la Revista Kepes, Grupo de estudio en Diseño Visual Fuente: elaboración propia, construido a partir de la página web de la revista ${ }^{6}$. 
a) Retícula Año 2 No. 1, revista KEPES 2005
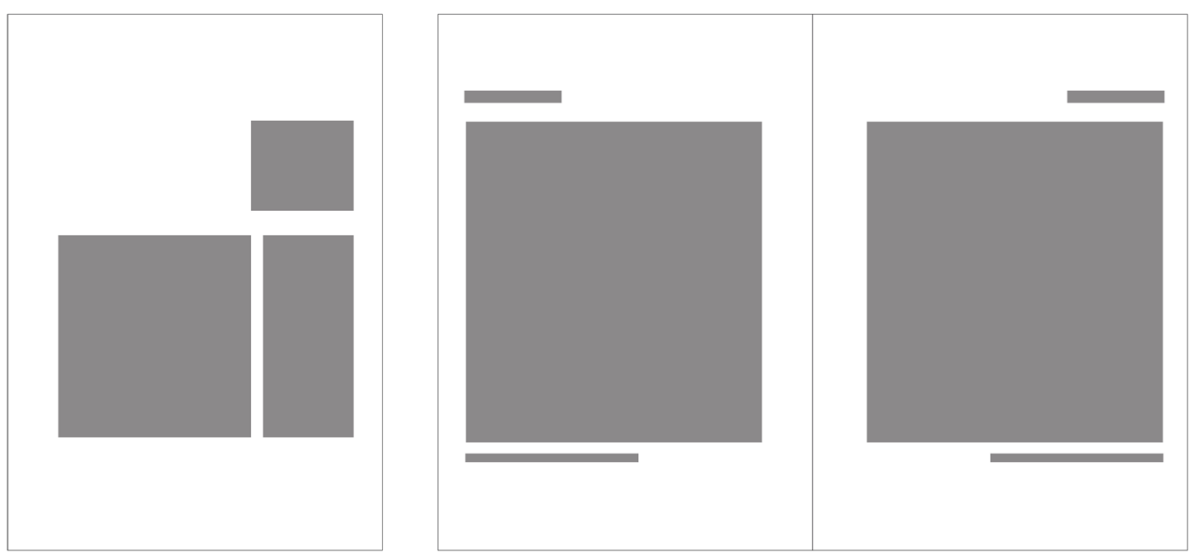

b) Año 3 No. 2, revista KEPES 2006 - 2019
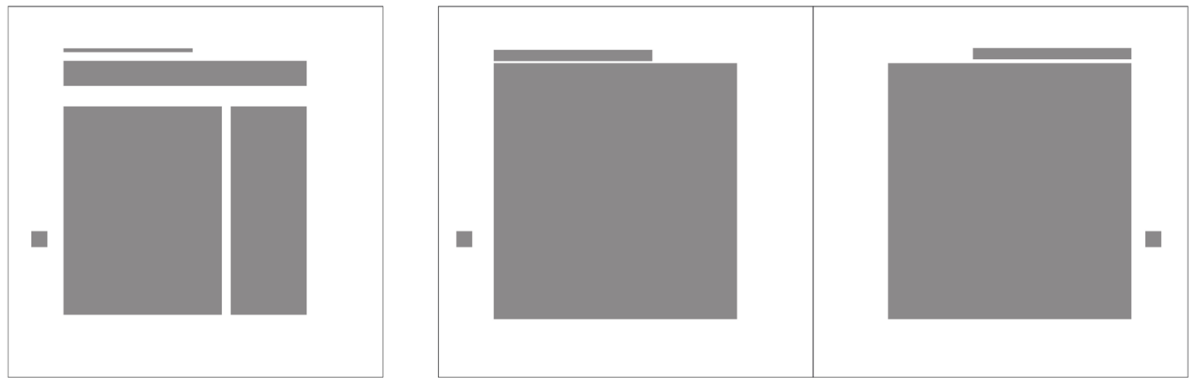

Figura 1. Comparativa de retículas Revista KEPES, Grupo de estudio en Diseño Visual. Fuente: elaboración propia. 


\section{El paso del logotipo a la simplificación de un signo}

El logotipo de KEPES (imagen 2) fue realizado por la Doctorando Claudia Jurado Grisales (Grupo de Estudios en Diseño Visual, 2004), quien se ha desempeñado como coordinadora editorial de la revista. La renovación del logo (imagen 2. B.) estuvo a cargo de la Diseñadora Visual Melissa Zuluaga Hernández bajo la dirección de la Doctorando Claudia Jurado. Actualmente Melissa es diseñadora y diagramadora de la Revista KEPES (2020).

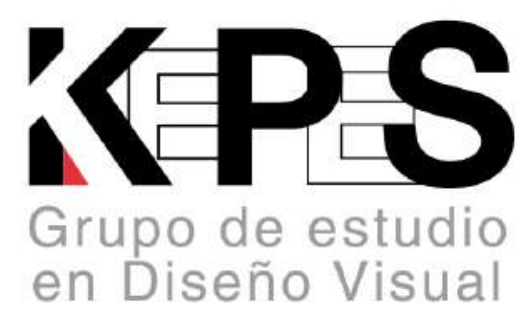

A- Logotipo Revista Kepes volumen 1 al 20 D. V. Claudia Jurado Grisales.

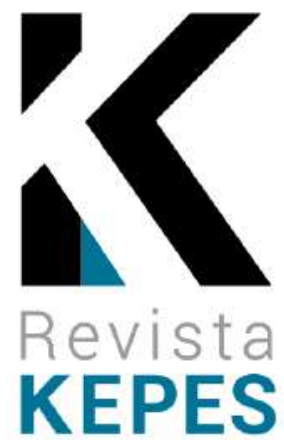

B- Logotipo Revista Kepes actualidad D. V. Melissa Zuluaga Hernández bajo la dirección de D. V. Claudia Jurado.

La marca KEPES es influenciada por el pensamiento de György Kepes, declarado mediante su libro "lenguaje de la visión" (Kepes et al., 1967), al igual que las características de la forma y la contraforma declaradas por la psicología de la Gestalt. 
Su texto se realiza con base en la fuente Neue Helvetica 75 Bold, realizando un juego de escalas tanto verticales como horizontales en las letras " $E$ ". Además de modificar el punto de curva inferior la letra S para el calce del remate con el brazo intermedio de la segunda " $\mathrm{E}$ ".

El ritmo de lectura se presenta en dos planos representado ligaduras bitonales (positivo/negativo). El primero constituido por las consonantes llenas (negras), mientras que las segundas las constituyen las vocales; las cuales son presentadas por contornos (blancas), ambas con espaciados al 125\%, sumado a un cuadrado entre las " $\mathrm{K}$ " el cual agrega el valor cromático a cada una de las ediciones (ver imagen 3).
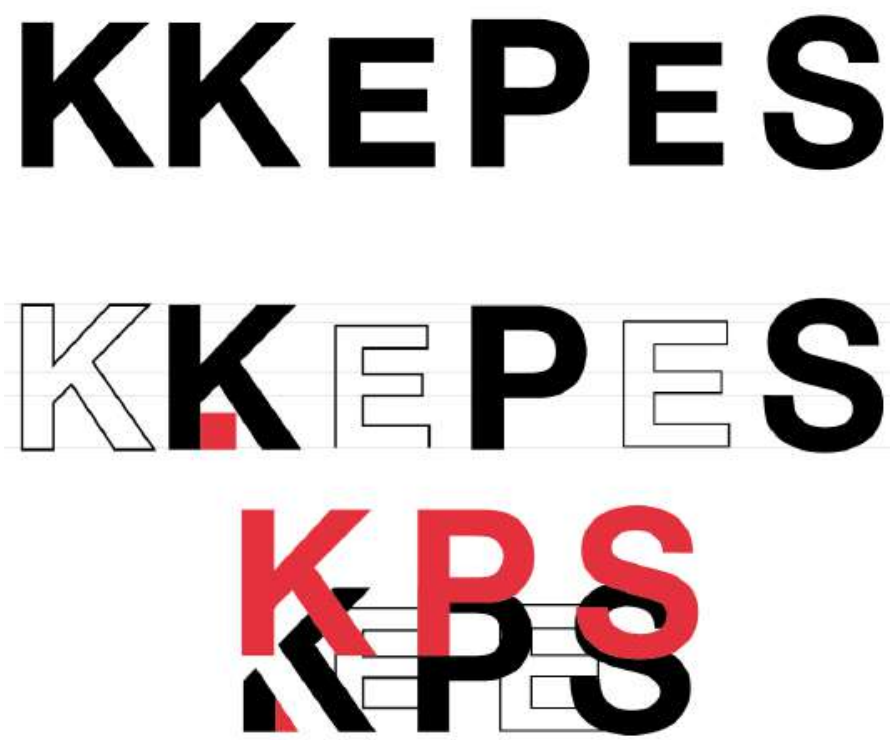

Imagen 3. Construcción del logotipo KEPES, Grupo de Estudio en Diseño Visual. Fuente: elaboración propia, construido a partir de entrevista a Claudia Jurado Grisales. 
Actualmente, por motivo del registro de marca, el logo ha sido modificado para darle valores diferenciadores por sobre los valores de lectura. Es entonces cuando, en la búsqueda de la prestancia y la identificación global de Kepes, la letra "K" pasa de un componente general del logotipo a tener el valor de SIGNO. El logo actual de la revista (ver imagen 2. B.) obtuvo su registro de marca mediante la Superintendencia de Industria y Comercio de Colombia, bajo la solicitud: SD2019/0107508, siendo publiado el: 18/02/2020.

\section{Indexación y políticas de KEPES}

La revista KEPES Grupo de estudio en Diseño Visual, se indexó a Publindex en 2006 (categoría c); proceso iniciado en 2005 por requerimiento de la Vicerrectoría de Investigaciones y Postgrados de la Universidad de Caldas (Londoño, 2005), generando un espacio ideal para la publicación, difusión, desarrollo e investigación científica tanto de manera general como específica al diseño visual en Colombia (Londoño, 2005). Luego, en $2011^{8}$ es indexada en las fuentes académicas Premiers-EBSCOhost - SCOPUS. Para la revista, estas indizaciones son una muestra de crecimiento y apoyo al almacenamiento, acceso expedito y libre a los contenidos. Además de asegurar un canal objetivo, en medio de la pluralidad conceptual y metodológica, característica del actual mundo científico (Villa, 2013).

A través de su web oficial: http://kepes.ucaldas.edu.co/, KEPES presenta una política de libre acceso a todo su registro histórico indexado ${ }^{9}$, lo que ayuda a la difusión libre y trasparente de la información. El libre acceso surgió entre fines del siglo XX y principios del siglo XXI como respuesta al creciente costo en

\footnotetext{
${ }^{7}$ https://sipi.sic.gov.co/sipi/Extra/IP/Mutual/Browse.aspx?sid=637321658856055517

${ }^{8}$ Coincidente con la celebración del vigésimo aniversario del Programa de Diseño Visual de la Universidad de Caldas, fundada en noviembre del 1991.

${ }^{9}$ Existen siete volúmenes (desde el 0 "cero" al 6) que no registran descarga directa en http://kepes.ucaldas.edu.co/, estos fueron proporcionadas de manera externa mediante el linkhttp://190.15.17.25/kepes/downloads/.
} 
las suscripciones a las revistas científicas, el cual era controlado por pequeños círculos académicos, haciéndolas totalmente inalcanzables al común de los investigadores (Galán et al., 2015; Ramírez-Godoy et al., 2019).

Se han generado campañas por medio de diferentes actores: académicos, científicos e instituciones, con el fin de que la ciencia y el conocimiento tenga libertad de flujo a toda la humanidad (Correa, 2018), no desconociendo de manera subyacente las grandes cifras que pagan anualmente las instituciones universitarias y centros de investigación por tener acceso a estas bases de datos.

Open Access 2020, por su lado, ha planteado nuevos desafíos, pretendiendo apertura y reutilización libre de los resultados de publicaciones científicas (Berlin Open Access Conference, 2018).

\section{Método}

El presente estudio incluyó el análisis de 269 documentos publicados por la revista KEPES entre 2004 y 2019, distribuidos en 16 volúmenes. La recopilación de los documentos se realizó por medio del sitio web de la propia revista. Los números 0 , 1, 2, 3 y 4 fueron recuperados de manera externa desde la misma base de datos alojada en el sitio web.

El método utilizado en este artículo es descriptivo (Montero \& León, 2007), pues se realiza un análisis del total de documentos, por medio de una descripción y categorización sistemática y metódica de indicadores generales como particulares, los cuales suministra la base de datos en la que se encuentra indexada la revista (tabla 1). 
En lo que concierne a la descripción de indicadores de colaboración y productividad, se usó el artículo como unidad básica de análisis. En la colaboración interinstitucional, se utilizaron las afiliaciones por autor en cada documento como criterio de evaluación, teniendo en cuenta, que un artículo puede tener autores de más de una institución.

A partir de estos datos se analizaron los diferentes indicadores, los cuales se repiten con mayor frecuencia en los diversos análisis cienciométricos y bibliometrías anteriormente citados. Generalmente, en los artículos se describe la cantidad de documentos con base en un conjunto determinado de variables y sus respectivas categorías (p. ej.:1, 2, 3, 4 autores, sexo, idioma, institución, tipo).

Por último y con el propósito de facilitar la descripción de los índices de colaboración y hacer evidente los resultados de manera clara, se han dividido los 16 años de trayectoria de la revista en pares.

\section{Plan de análisis}

Tabla 1. Indicadores analizados en el artículo

\begin{tabular}{lc}
\hline \multicolumn{1}{c}{ Nombre del indicador } & Tabla de indicadores analizados en este artículo \\
\hline \multicolumn{2}{l}{ Indicadores de tipo bibliométricos } \\
\hline Tipo de documentos & Totalidad de documentos en la revista entre los años 2004-2018. \\
\hline Idioma & Corresponde al tipo de publicación, de acuerdo con la clasificación por jueces. \\
\hline Institución & Corresponde al idioma en que está escrito el corpus de la publicación. \\
\hline País & Nombre de la institución asociada informada por cada uno de los autores. \\
\hline Sexo & Nacionalidad de la institución asociada a cada uno de los autores. \\
\hline
\end{tabular}




\begin{tabular}{|c|c|}
\hline Nombre del indicador & Descripción del indicador \\
\hline & Indicadores de tipo colaboración \\
\hline Número de autores & Totalidad de autores informados en la publicación. \\
\hline \multirow{4}{*}{ Índice de Lawani } & $\begin{array}{l}\text { Concierne a la media de autores por documentos, distribuidos por los periodos } \\
\text { de publicación. Se calcula mediante la siguiente ecuación: }\end{array}$ \\
\hline & Índice de Lawani $=\frac{\sum_{i=1}^{N} j_{i} n_{i}}{N}$ \\
\hline & $\begin{array}{l}\text { donde: } \\
\boldsymbol{N}=\text { Número de documentos }(\text { Lawani, 1981; Vinkler, 1993:224). } \\
\boldsymbol{j i}=\text { Frecuencia de (i) coautores en colaboración. } \\
\boldsymbol{n j}=\text { Número de documentos }(j) \text { en colaboración por i coautores. }\end{array}$ \\
\hline & No hay un valor máximo para este índice. \\
\hline \multirow{6}{*}{ Índice de Subramanyam } & $\begin{array}{l}\text { Concierne a la cantidad de documentos con múltiple autoría. Es calculado por } \\
\text { medio de la siguiente ecuación: }\end{array}$ \\
\hline & Índice de Subramanyam $=\frac{\mathrm{Nm}}{\mathrm{Nm}+\mathrm{Nc}}$ \\
\hline & donde. \\
\hline & $0 \leq$ Índice de Subramanyam $\leq 1$ \\
\hline & $\begin{array}{l}\mathrm{Nm}=\text { Número de documentos con autoría múltiple publicados, durante un } \\
\text { periodo determinado. }\end{array}$ \\
\hline & $\begin{array}{l}\text { Ns = Número de documentos con autoría única en el mismo periodo } \\
\text { determinado (Subramayan \& Stephens, 1982; Subramanyam, 1983: 37). }\end{array}$ \\
\hline \multirow{6}{*}{ Coeficiente de colaboración } & $\begin{array}{l}\text { Concierne a la proporción de artículos con autoría múltiple ( } 2 \text { o más autores). } \\
\text { Se calcula por medio de la siguiente ecuación: }\end{array}$ \\
\hline & Coeficiente de Colaboración $=\frac{\sum_{i=1}^{N}\left(\frac{1}{j i}\right) n i j}{\mathrm{~N}}$ \\
\hline & donde: \\
\hline & $N=$ Número de documentos. \\
\hline & $\begin{array}{l}\boldsymbol{J} \boldsymbol{i}=\text { Frecuencia de } \boldsymbol{i} \text { coautores en colaboración. } \\
\text { nij=Número de documentos ( } \mathrm{j} \text { ) en colaboración por (i) coautores. }\end{array}$ \\
\hline & $\begin{array}{l}\text { Cuyo valor oscila igual que el anterior entre } 0 \text { y } 1 \text {, por lo que valores obtenidos } \\
\text { cercanos a } 0 \text { indican una gran cantidad de documentos de un solo autor } \\
\text { (Ajiferuke, Burell, \& Tague, 1988, p. 424) }\end{array}$ \\
\hline
\end{tabular}




\begin{tabular}{|c|c|}
\hline Nombre del indicador & Descripción del indicador \\
\hline \multicolumn{2}{|r|}{ Indicadores de relación } \\
\hline Participación autores totales & $\begin{array}{l}\text { Concierne al número de autores (por nacionalidad o institución) que conforman } \\
\text { la totalidad de los artículos sin discriminación de su posición. }\end{array}$ \\
\hline Primera autoría & $\begin{array}{l}\text { Concierne al número de autores (por nacionalidad o institución) que poseen la } \\
\text { primera posición en la totalidad de los artículos. }\end{array}$ \\
\hline Primera autoría solo & $\begin{array}{l}\text { Concierne al número de autores (por nacionalidad o institución) que participan } \\
\text { solos en una publicación en la totalidad de los artículos. }\end{array}$ \\
\hline \multirow{4}{*}{ Interrelación } & $\begin{array}{l}\text { Concierne al número de autores que participan con misma nacionalidad o } \\
\text { institución en una publicación. }\end{array}$ \\
\hline & Inter - relación $=\frac{\sum_{i=1}^{N}\left(n_{i} \equiv f\right)-\left(n_{i} \neq f\right)}{N}$ \\
\hline & $\begin{array}{l}\text { donde: } \\
\boldsymbol{N}=\text { Número de autores en el artículo. } \\
\boldsymbol{f}=\text { Institución o país del primer autor } \\
\boldsymbol{n} \boldsymbol{i}=\text { Número de autores }\end{array}$ \\
\hline & $\begin{array}{l}\text { Cuyo valor oscila entre } 0 \text { y } 1 \text {. El valor } 1 \text { denota participación nacional y } \\
\text { cualquier número menor a } 1 \text { indica participación internacional } \\
\text { (Uribe-Bahamonde \& Díaz Herrera, 2019). }\end{array}$ \\
\hline Número de relaciones & $\begin{array}{l}\text { Concierne a la relación existente entre el primer autor y el resto de los autores } \\
\text { del mismo artículo de países o instituciones diferentes. }\end{array}$ \\
\hline
\end{tabular}

Fuente: elaboración propia.

\section{Resultados}

Los resultados consideran tres características esenciales: la productividad, la revista y su evolución en el tiempo desde su fundación. Además, se exhiben indicadores bibliométricos, colaboración, coeficiente de colaboración e indicadores de relación.

La figura 2 hace evidente que KEPES presenta una constitución netamente ensayística, la cual es predominante en toda la vida de la revista. No obstante, 
se observa el inicio de la publicación de artículos científicos en 2007. Posterior a la fecha, estos han aumentado, obteniendo los índices más altos en el 2015, para luego consolidarse en 2019.

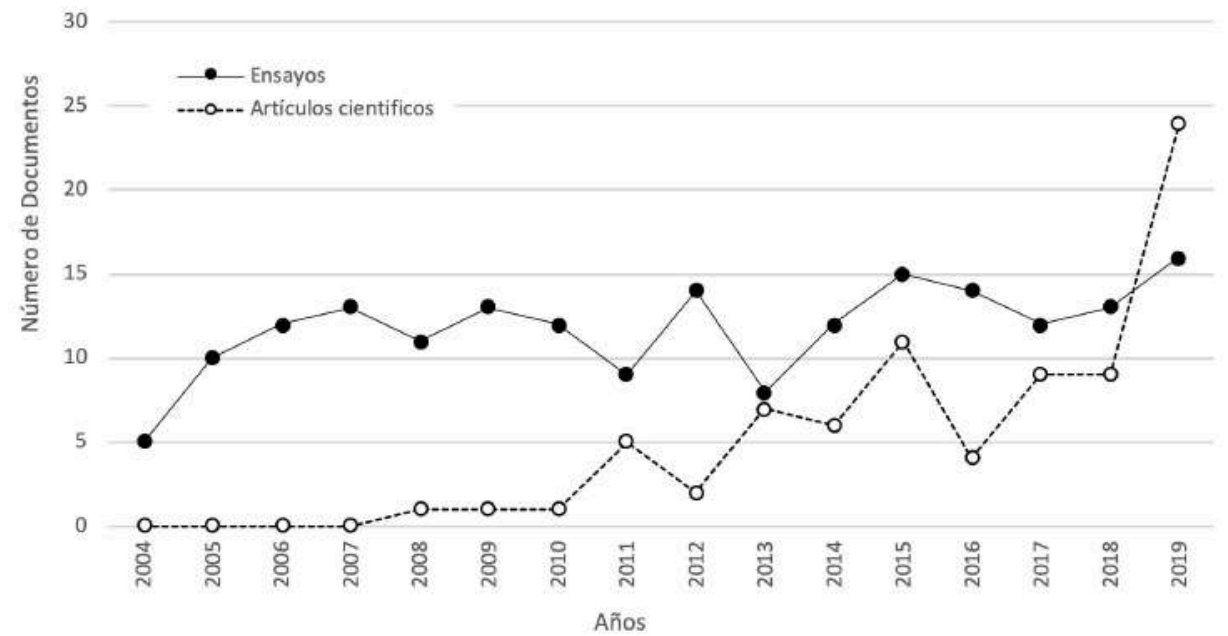

Figura 2. Indicadores de número de documentos por año de publicación diferenciados por tipo de documento Número de documentos elaborados como: ensayos o artículos científicos. Fuente: elaboración propia.

La figura 3 da cuenta de la primacía del idioma español en la estructura de la revista. La participación de documentos en inglés aumenta en 2006 y 2012. Este tipo de documentos no se sostiene en el tiempo. Con respecto al idioma portugués, este se presenta como un dato atípico en 2007, al igual que un documento en italiano en 2012. 


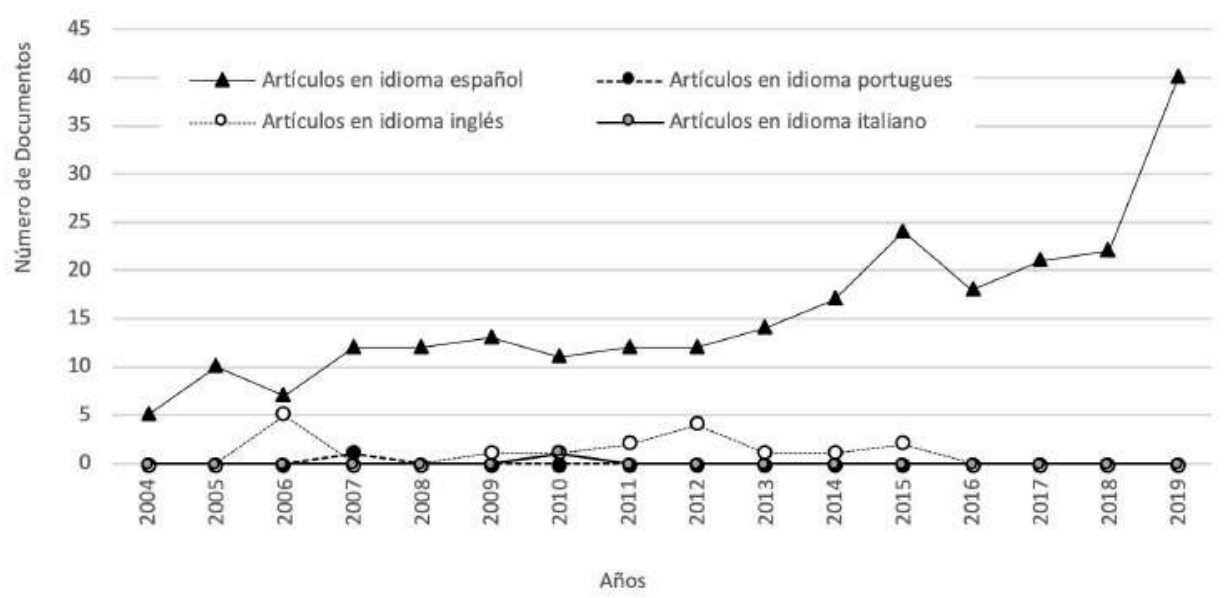

Figura 3. Indicadores de número de documentos escritos por año de publicación diferenciados por idioma. Número de apariciones de los idiomas: español, inglés, portugués e italiano. Fuente: elaboración propia.

La figura 4 evidencia la participación de hombres y mujeres por año, destaca una importante diferencia en la participación de mujeres desde los años de su fundación (Kepes 0 "cero", año 2004). Esta se mantiene constante hasta 2009 en todas las participaciones. En 2010, se ve su primer crecimiento y estrechamiento en relación con la participación de hombres. En 2012 se equiparará la participación de hombres y mujeres, hasta 2013, luego vuelve a distanciar su participación para aproximarse nuevamente en 2018.

El recuento total de autorías masculinas y femeninas (2004-2019), podemos encontrar 244 participaciones de hombres correspondientes al $63 \%$ del volumen total de autorías de artículos, mientras que las mujeres tienen participación en un total de 143 autorías equivalentes al 37\% de la muestra final. 


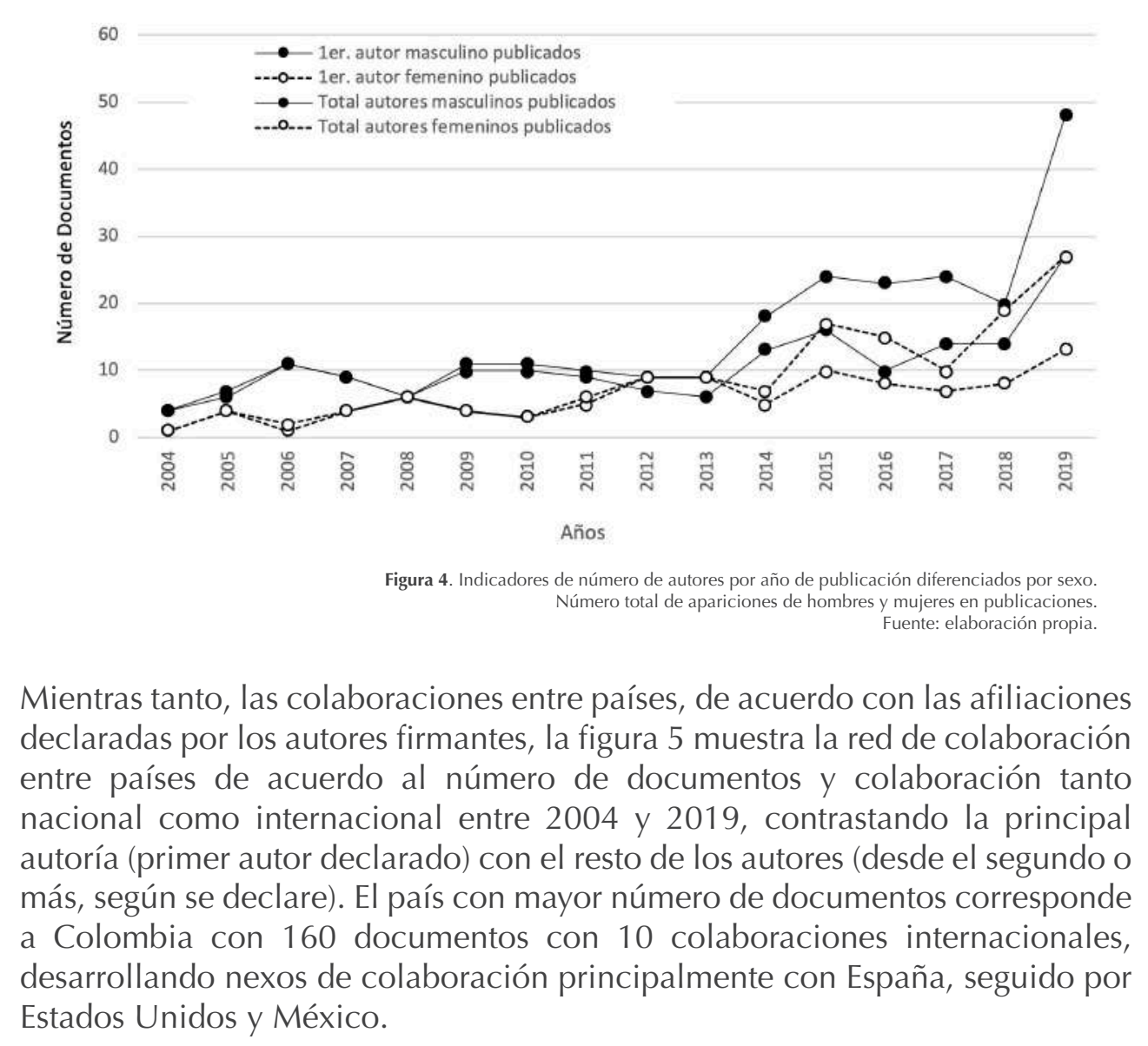




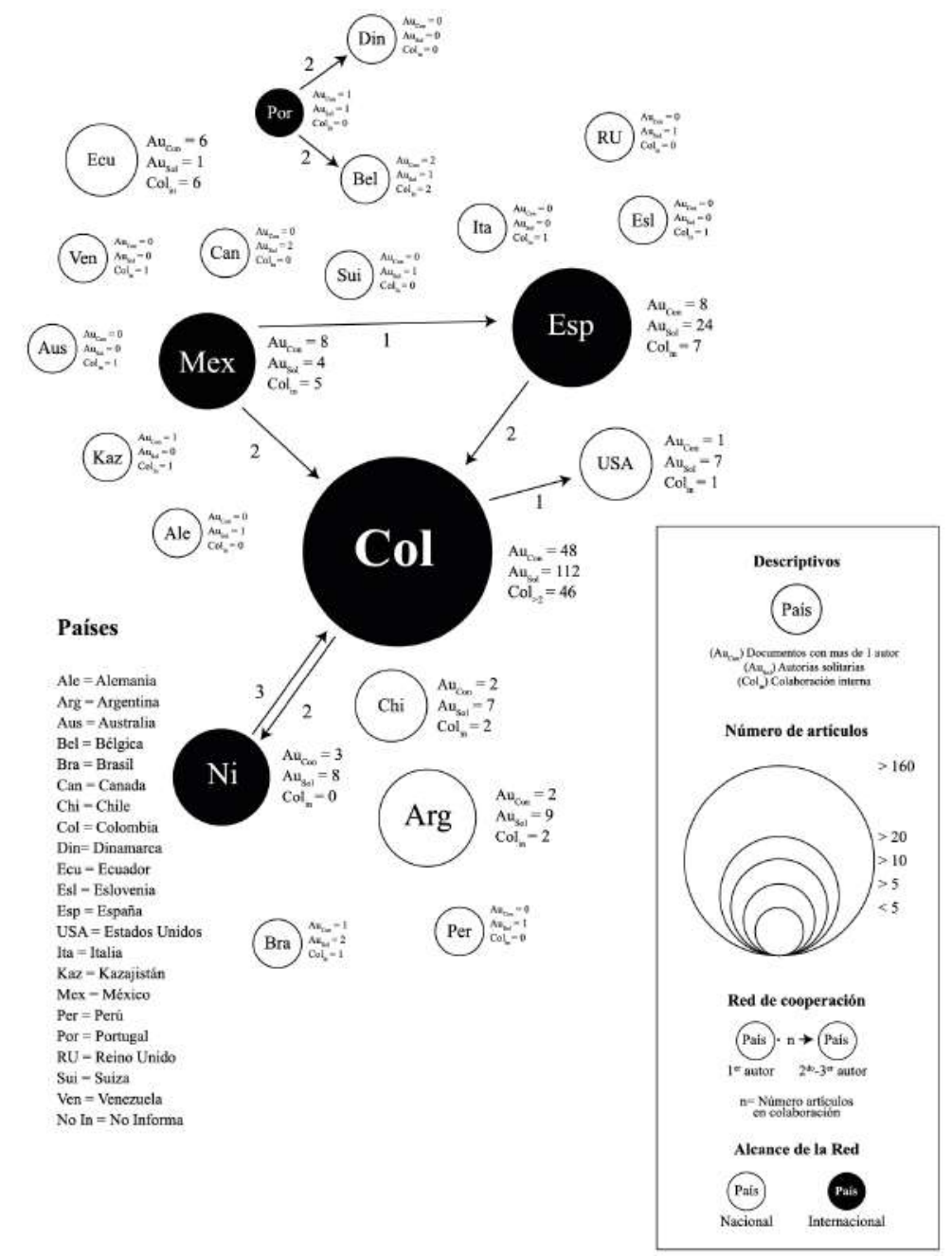

Figura 5. Mapa de colaboración entre instituciones por medio de artículos publicados en revista KEPES entre 2004-2019. Fuente: elaboración propia. 
La figura 6 muestra el mapa de colaboración entre instituciones de acuerdo con la cantidad de documentos y colaboración entre 2004-2019, contrastando la principal autoría (primer autor declarado) con los coautores (desde el segundo o más, según se evidencie).

La institución con mayor número de documentos corresponde a la Universidad de Caldas con 11 colaboraciones interinstitucionales, seguido por las universidades Autónoma de Aguascalientes y Nacional de Colombia; en menor medida la Universidad Católica de Manizales, Universidad Tecnológica de Pereira y Pontificia Universidad Javeriana. Son destacables los nexos creados por la Universidad Nacional de Colombia, quién desarrolla lazos con Fundación Universitaria Los Libertadores y el Instituto Tecnológico Metropolitano.

El desarrollo de colaboraciones por medio de la institución representante de la editorial presenta elementos pasivos de participación. Esta se desarrolla principalmente, de manera individual, en contraposición a lo realizado por la Universidad Nacional de Colombia, que no presenta documentos de manera independiente.

La mayor participación en la revista comprende a la Universidad de Caldas, con 56 documentos, seguida por la Universidad Pontificia Bolivariana con 18 documentos y la Universidad Nacional de Colombia con 15 documentos.

Con respecto a los autores con más participaciones en KEPES, destaca la participación de Walter Castañeda y Felipe Londoño, quienes se enfrentan en dinámicas contrarías al trabajo individual versus trabajo colaborativo respectivamente. 


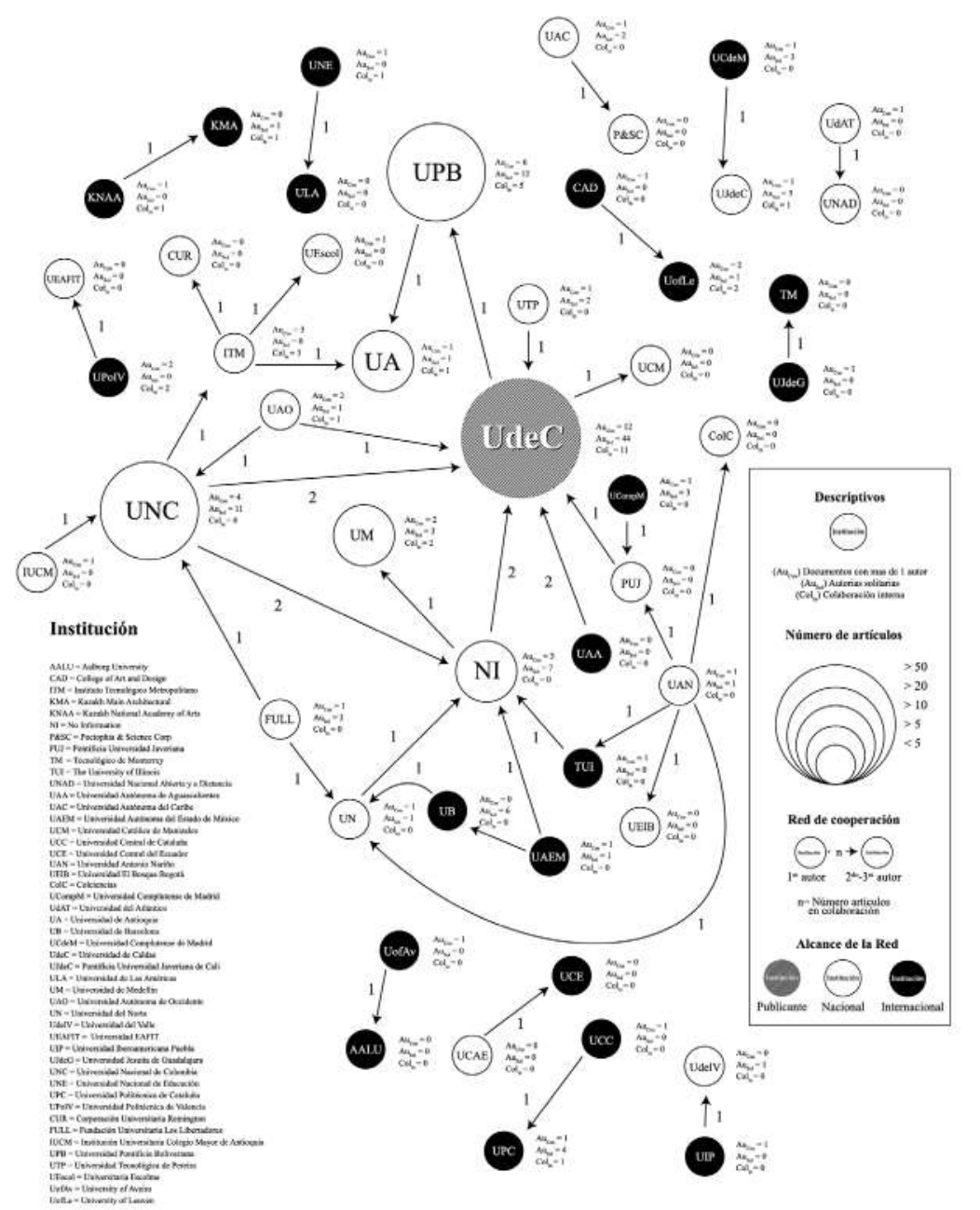

Figura 6. Mapa de colaboración entre países a partir de los artículos publicados en revista KEPES entre 2004-2019. Fuente: elaboración propia. 
Uribe, Y. / KEPES, desde Colombia al mundo. Una tarea pendiente: análisis bibliométrico y relacional.

Los nueve autores más destacados de la revista KEPES y su número de publicaciones se resumen en la tabla 2.

Tabla 2. Lista de los nueve mayores publicantes de la revista KEPES entre 2004-2019, diferenciados por posición de autoría y tipo de colaboración en sus respectivas publicaciones

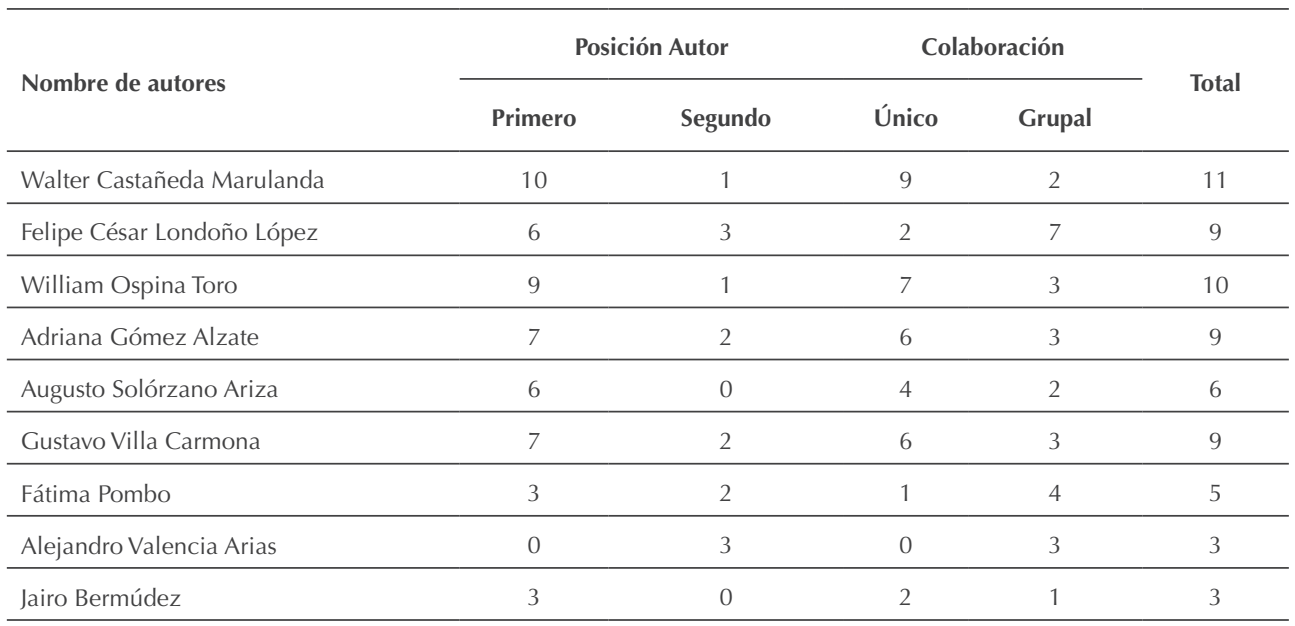

Fuente: elaboración propia.

A partir de la tabla 1, se ha elaborado la figura 7, detectando y rastreando las conexiones de dichos autores con sus diversos coautores. El mapa de colaboración deja en evidencia 3 niveles de autorías: internos, propios de la Universidad de Caldas; externos, autores de afiliación colombiana y, por último, las autorías internacionales, compuesta por autores de afiliación externa a Colombia. 
En la figura se exponen diversos grupos de investigación, el principal de ellos es el proyectado por Felipe César Londoño López en un eje correspondiente a la Universidad de Caldas, seguido por un segundo núcleo de la Universidad de Leuven, liderado por Fátima Pombo.

Es importante resaltar el hermetismo desarrollado por parte del Grupo de Estudio visual de la Universidad de Caldas. En ninguno de sus artículos donde figuran como primer autor, agregan a un investigador externo al grupo. Por lo que su participación en colaboraciones inter-institucional se hace presente solo como un apoyo a las investigaciones publicadas. 


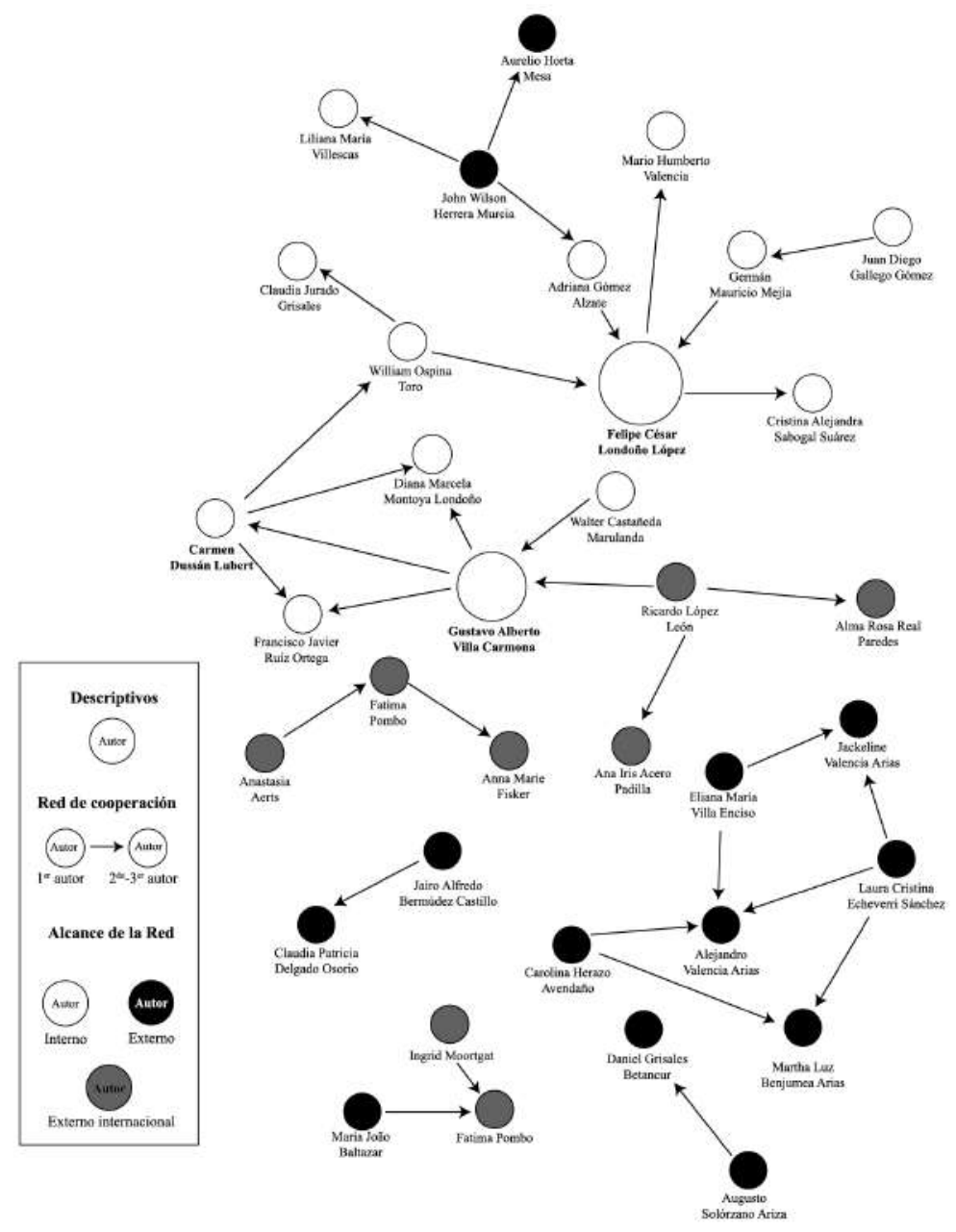

Figura 7. Mapa de colaboración entre mayores autores por medio de artículos publicados en revista KEPES entre 2004-2019. Fuente: elaboración propia. 
La tabla 2 muestra la cantidad de autores por producción científica. El mayor porcentaje de trabajos $(70 \%)$ es presentado por solo un autor, mientras que la producción de autoría múltiple posee un porcentaje significativamente menor (30\%). Comparando los periodos de extremos: primer periodo (2004-2005) y las del último (2018-2019), se observa una diferencia entre la cantidad de documentos en los mismos periodos de tiempo. En términos porcentuales, la muestra indica que la cantidad de documentos recibidos por año se ha duplicado (de 15 a $62=403 \%$ ). La participación de autores se redistribuye, aumentando de $6,7 \%$ a $48 \%$ en el caso de la autoría múltiple, mientras que la autoría individual presenta una disminución desde el 93,3\% hasta un 52\%. El índice Subramanyam nos reafirma el crecimiento de la participación de autoría múltiple, desde un 0,07 en el periodo 2004-2005 hasta un 0,48 en el periodo 2018-2019. Además, se presenta el índice de Lawani, el cual indica que junto al crecimiento de documentos con autoría múltiple, el aumento de colaboración se hace evidente en los últimos tres periodos, pasando de 0,32 colaboradores por artículo en el periodo 2012-2013 a 1,49 colaboradores por artículo en el periodo 2016-2017.

Al unir los índices de Subramanyam y Lawani, ha aumentado la cantidad de autores firmantes por artículo al igual que la cantidad de artículos en los que se presentan dos o más autores, reafirmando lo declarado por el coeficiente de colaboración. 
Tabla 3. Número de documentos de la revista KEPES agrupados según cantidad de autores y diferenciados por periodo

\begin{tabular}{|c|c|c|c|c|c|c|c|c|c|c|c|c|c|c|c|c|c|c|}
\hline \multirow{3}{*}{$\begin{array}{c}\begin{array}{c}\text { Número } \\
\text { de } \\
\text { autores }\end{array} \\
1\end{array}$} & \multicolumn{18}{|c|}{ Período } \\
\hline & \multicolumn{2}{|c|}{ 2004-2005 } & \multicolumn{2}{|c|}{ 2006-2007 } & \multicolumn{2}{|c|}{ 2008-2009 } & \multicolumn{2}{|c|}{$2010-2011$} & \multicolumn{2}{|c|}{$2012-2013$} & \multicolumn{2}{|c|}{ 2014-2015 } & \multicolumn{2}{|c|}{ 2016-2017 } & \multicolumn{2}{|c|}{ 2018-2019 } & \multicolumn{2}{|c|}{ TOTAL } \\
\hline & 14 & $93,3 \%$ & 24 & $96 \%$ & 25 & $96 \%$ & 24 & $89 \%$ & 26 & $84 \%$ & 30 & $68 \%$ & 13 & $33 \%$ & 32 & $52 \%$ & 188 & $70 \%$ \\
\hline 2 & 1 & $6,7 \%$ & 1 & $4 \%$ & 1 & $4 \%$ & 3 & $11 \%$ & 5 & $16 \%$ & 8 & $18 \%$ & 22 & $56 \%$ & 16 & $26 \%$ & 57 & $21 \%$ \\
\hline 3 & 0 & $0 \%$ & 0 & $0 \%$ & 0 & $0 \%$ & 0 & $0 \%$ & 0 & $0 \%$ & 4 & $9 \%$ & 2 & $5 \%$ & 10 & $16 \%$ & 16 & $6 \%$ \\
\hline 4 & 0 & $0 \%$ & 0 & $0 \%$ & 0 & $0 \%$ & 0 & $0 \%$ & 0 & $0 \%$ & 2 & $5 \%$ & 2 & $5 \%$ & 2 & $3 \%$ & 6 & $2 \%$ \\
\hline$>4$ & 0 & $0 \%$ & 0 & $0 \%$ & 0 & $0 \%$ & 0 & $0 \%$ & 0 & $0 \%$ & 0 & $0 \%$ & 0 & $0 \%$ & 2 & $3 \%$ & 2 & $1 \%$ \\
\hline Total & & 15 & & 25 & & 26 & & 7 & & 31 & & 44 & & 39 & & 62 & & 69 \\
\hline \multicolumn{19}{|l|}{ Índices } \\
\hline $\mathrm{GC}^{\mathrm{a}}$ & \multicolumn{2}{|c|}{0,07} & \multicolumn{2}{|c|}{0,04} & \multicolumn{2}{|c|}{0,04} & \multicolumn{2}{|c|}{0,11} & \multicolumn{2}{|c|}{0,16} & \multicolumn{2}{|c|}{0,32} & \multicolumn{2}{|c|}{0,67} & \multicolumn{2}{|c|}{0,48} & \multicolumn{2}{|c|}{0,24} \\
\hline $\mathrm{IC}^{\mathrm{b}}$ & \multicolumn{2}{|c|}{0,13} & \multicolumn{2}{|c|}{0,08} & \multicolumn{2}{|c|}{0,08} & \multicolumn{2}{|c|}{0,22} & \multicolumn{2}{|c|}{0,32} & \multicolumn{2}{|c|}{0,82} & \multicolumn{2}{|c|}{1,49} & \multicolumn{2}{|c|}{1,13} & \multicolumn{2}{|c|}{0,53} \\
\hline $\mathrm{CC}^{\mathrm{c}}$ & \multicolumn{2}{|r|}{0,03} & \multicolumn{2}{|c|}{0,02} & \multicolumn{2}{|c|}{0,02} & \multicolumn{2}{|c|}{0,06} & &, 09 & &, 23 & & 0,55 & & 41 & & 18 \\
\hline
\end{tabular}

Nota: los porcentajes se calcularon de manera independiente a cada periodo.

a Índice Subramanyam obtenido mediante el periodo total (2004-2019)

b Índice Lawani obtenido mediante el periodo total (2004-2019)

c Coeficiente de Colaboración (cc)

Fuente: elaboración propia.

\section{Conclusiones}

KEPES nace del interés de externalización de discusiones planteadas dentro del grupo de estudio en diseño visual de la Universidad de Caldas, con el objetivo de generar nuevas reflexiones en torno al diseño a nivel institucionallocal, y cuya expectativa de crecimiento y proyección se ve enfatizada por la Vicerrectoría de Investigaciones y Postgrados de la Universidad de Caldas.

Una de las características que impacta directamente en la conducción de la revista es su composición multi e interdisciplinaria, afectando directamente en el tipo de documentos que componen la fundación de esta —ensayística— 
y se contrapone con el paradigma cientificista de indexación y rigurosidad empírica-fáctica del medio.

Si bien el idioma principal de la comunicación científica es el inglés, KEPES no pierde su foco de ampliar el conocimiento del diseño por medio del idioma español, este factor es bastante interesante al situarse junto a la revista chilena "Boletín del Museo Chileno de Arte Precolombino" en lo más alto del ranking temático "Visual Arts and Performing Arts" de SCIMAGO.

La participación de la mujer en la ciencia ha presentado un nuevo foco en las temáticas de equidad de género, KEPES — como revista científica- no escapa de esta crítica en su fundación, evidenciándose como una revista principalmente masculina, no obstante, en su recorrido histórico ha equiparado su participación con lo que puede considerarse una distribución normal en el campo, no obstante, al comparar la colaboración entre hombres / mujeres, los hombres colaboran un $74 \%$ con otros hombres y solo un $26 \%$ con autoras mujeres, mientras que las mujeres con primera autoría, trabajan de manera equilibrada entre hombres (53\%) y mujeres (46\%).

KEPES inicialmente presentaba altos niveles de endogamia (característico de revistas científicas artesanales), factor totalmente criticable y cuestionable en procesos de indización. No obstante, se plantean como recursos válidos en el puntapié organizacional de las revistas, siempre que estas prácticas se eliminen en el paso del tiempo, fenómeno que la revista sortea loablemente.

Las vinculaciones por parte de la institución editora, a través de sus publicaciones, demarca una falencia integradora — en autorías cooperantescosa que sí es aprovechada por la Universidad Nacional de Colombia. Este elemento es corroborado al seguir las conexiones de los autores con mayor cantidad de documentos. 
Un factor primordial en la falta de vinculación temprana es la constitución de los ensayos y el trabajo departamental. Mientras el ensayo es determinado por pensamientos y criticas personales, el departamental es guiado íntegramente por un líder de investigación.

Al dejar de lado la ensayística e integrar la dinámica del artículo científico instaura procesos integradores de construcción empírica y replicable. Determinante claro de la posibilidad de trabajo en equipo, bajo un pensamiento unificado, haciendo que aumente el número de autores firmantes y con ello, se evidencia un aumento en los grados de colaboración, no obstante, los numero de colaboración son por intrínsecos y no distantes, con lo que la generación de grupos de estudios se plantea de maneras disgregadas los propios saberes de estudio.

Los índices de colaboración han aumentado notablemente en el último periodo de tiempo (2014-2018), y entregan real evidencia del gran trabajo editorial, por otro lado, es importante destacar que este aumento en la cantidad de autores pueda explicarse por la correlación correspondiente a un r2 =0,997, al correlacionar el "índice de Lawani" con el "índice de impacto" de la revista por medio de SCIMAGO durante los años 2011-2019. A mejor posicionamiento en el ranking de indización, mayor cantidad de autores.

La Universidad de Caldas, en su calidad de "casa editorial", no ha podido sacar provecho investigativo-colaborativo y ha centrado sus energías en la elaboración de líneas investigativas propias al grupo. A diferencia de la Universidad Nacional de Colombia, la cual genera mayor variedad de vínculos interinstitucionales, explicado por su calidad de universidad compleja y su actual posición 259 en el ranking mundial de universidades declarado por Top Universities ${ }^{10}$.

${ }^{10} \mathrm{https} / / /$ www.topuniversities.com/universities/universidad-nacional-de-colombia \#wurs 
Parece relevante destacar que estos estudios, inspirados en la colaboración y producción del conocimiento científico, logran expresar un diagnóstico longitudinal de la producción científica de manera cuantitativa y que por lo cual, puede analizarse como mecanismo de gestión editorial para la toma de futuras decisiones. Ahora bien, qué duda cabe que el crecimiento de KEPES se concatena con la relevancia de la indexación, la cual ha aumentado en impacto, categoría, citación y colaboraciones a presentar en la revista, otorgándole un lugar de relevancia dentro de las artes visuales y humanidades, siendo la segunda revista latinoamericana y vigésimo primera a nivel mundial en el área ${ }^{11}$. Un espacio de legitimación académica internacional, estatus que se expresa una vez que logra su indización a SCOPUS. Quizás, los datos expuestos en el análisis puedan ser vistos como negativos (endogamia académica, grupos de estudios cerrados, exposición de la ensayística por sobre los artículos científicos), están más lejos de aquello, ya que se presentan como una serie de oportunidades para la mejora y la generación de mayores vínculos interinstitucionales, que irán en ayuda a la perfección cualitativa y colaborativa de la propia revista KEPES y su grupo de estudio visual.

\section{La necesidad (i?) de sumarse a la modernización de la gestión en ciencia, tecnología y academia}

El Grupo de Estudio en Diseño Visual, en voz de Felipe Londoño López, asume que el nacimiento estandarizado de la Revista KEPES está condicionado a la necesidad de "abrir un espacio para la publicación, difusión e investigación científica en torno al Diseño Visual en Colombia". Sin embargo, el requerimiento de indización por parte de la Vicerrectoría de Investigaciones y Postgrados de la Universidad de Caldas, busca dar respuesta al proyecto de COLCIENCIAS. El cual, desde el año 2000, cimenta su interés de modernizar las instituciones. Esto para aumentar, por medio del conocimiento, la producción científica, 
tecnológica y la innovación y así, a través de "grupos de estudios", ubicar a Colombia entre los países más innovadores de América latina.

Pero, ¿qué implica modernizar las instituciones? Involucra asimilar procesos de gestión y administración, en los cuales emerge el fortalecimiento del mercado y su liberalización, con el objeto de estimular la competencia en el mercado de bienes y servicios (Escobar-Espinoza \& Mercado-Mejía, 2014). Esto nos invita a reflexionar críticamente respecto a la institucionalidad y sus condiciones modernizadoras; de entender la generación del conocimiento a través de las diferentes formas de expresar la producción científica.

Podemos así asumir la crítica estipulada por (Irizar et al., 2015), en relación a la pretensión de dominación de esta técnica analítica cienciométrica que ha primado en el último tiempo bajo cánones "perversos". Es por ello, que se instala la práctica de asumir institucionalmente ciertas políticas propensas a la medición, objetualización, con juicios de calidad generalizados y estandarizados, lo que determinan conductas y condicionan el quehacer propio de la editorial, construyendo y dando forma a valores característicos y que pueden llegar a entrar en crisis con los diversos escenarios, a nivel estructural de las propias instituciones (Díaz, 2018).

Por otro lado, según Ordorika ( 2018) las mediciones bibliométricas forman parte de este cúmulo de carrera académica, en relación a la primacía del mercado que termina por determinar forma del Estado, la sociedad y la cultura; así como la adopción de un lenguaje propio del mercado, la competitividad, rentabilidad, eficiencia, etc. que terminan por validar la ciencia. Según lo anterior, parece relevante estos estudios que, inspirados en la circulación y producción del conocimiento científico, logran expresar por una parte un diagnóstico longitudinal de la producción científica que puede analizarse como mecanismo de gestión editorial para la toma de decisiones. 
Sin embargo, este tipo de trabajos invita a reflexionar respecto a la necesidad de sumarse a estas lógicas mercantiles de producción, que si lo pensamos dos veces está alentando un capitalismo académico, que por lo visto, está lejos de retroceder limitando el énfasis emancipador de la construcción del conocimiento.

He aquí la invitación de esta autora generar un trabajo que coadyuve a la gestión y toma de decisiones de la editorial de la revista y además de ser un firme contrapunte a la realización posterior de un estudio temático cuantitativo en búsqueda del ethos propio de la revista KEPES.

\section{Agradecimientos}

El autor de este artículo agradece encarecidamente la facilitación de documentos e información fundacional no disponible en línea realizado por el profesor Gustavo Villa Carmona († 22 de julio de 2019), quien lamentablemente falleció mientras se realizaba este estudio; además, a la gestión y facilitación de información actualizada de la revista por parte del Dr. Walter José Castañeda Marulanda y finalmente, pero no por ello menos importante, a la doctorando Claudia Jurado Grisales, quien ha facilitado mediante una entrevista, datos no documentados de la línea gráfica de la revista KEPES desde su fundación. 


\section{Referencias}

Aguillo, I. F., Granadino, B., Ortega, J. L., \& Prieto, J. A. (2006). Scientific research activity and communication measured with cybermetrics indicators. Journal of the American Society for Information Science and Technology, 57(10), 1296-1302. DOI: https://doi.org/10.1002/asi.20433

Ajiferuke, I., Burell, Q., \& Tague, J. (1988). Collaborative coefficient: A single measure of the degree of collaboration in research. Scientometrics, 14(5-6), 421-433. DOI: https://doi.org/10.1007/BF02017100

Araújo,J.yArencibia, R.(2002).Infometría, bibliometría y cienciometría:aspectos teórico - prácticos. Acimed, 10(4), 4040. Recuperado de http://scielo.sld. cu/scielo.php?script=sci_arttext\&pid=S1024-94352002000400004

Aravena, P., Cartes-Velásquez, R. y Manterola, C. (2013). Productividad y calidad metodológica de artículos clínicos en cirugía oral y maxilofacial en Chile: Período 2001-2012. Revista Chilena de Cirugía, 65(5), 382-388. DOI: https://doi.org/10.4067/S0718-40262013000500003

Bailón-Moreno, R., Jurado-Alameda, E., Ruiz-Baños, R., \& Courtial, J. P. (2005). Bibliometric laws: Empirical flaws of fit. Scientometrics, 63(2), 209-229. DOI: https://doi.org/10.1007/s11192-005-0211-5

Berlin Open Access Conference. (2018). Report, Statements and Press 14th Berlin Open Access Conference. Recuperado de https://oa2020.org/wpcontent/uploads/pdfs/14thBerlinOpenAccessConference_Report.pdf

Björk, B. C., Laakso, M., Welling, P., \& Paetau, P. (2014). Anatomy of green open access. Journal of the Association for Information Science and Technology, 65(2), 237-250. DOI: https://doi.org/10.1002/asi.22963 
Björneborn, L., \& Ingwersen, P. (2004). Toward a basic framework for webometrics. Journal of the American Society for Information Science and Technology, 55(14), 1216-1227. DOI: https://doi.org/10.1002/asi.20077

Braun, T., Glänzel, W., \& Schubert, A. (1988). The newest version of the facts and figures on publication output and relative citation impact of 100 countries 1981-1985. Scientometrics, 13(5-6), 181-188. DOI: https://doi. org/10.1007/BF02019957

Cartes-Velásquez, R., Moraga, J., Aravena, P. y Manterola, C. (2012). Impacto y visibilidad de la Revista Chilena de Cirugía tras su indización en las bases de datos SciELO e ISI: Análisis bibliométrico. Revista Chilena de Cirugía, 64(6), 511-515. DOI: https://doi.org/10.4067/S0718-40262012000600003

Correa, J. D. C. (2018). Reflexiones en torno a la arqueología de medios en el software libre. KEPES, 15(17), 69-92. DOI: https://doi.org/10.17151/ kepes.2018.15.17.4

Cudina, J. N., \& Ossa, J. C. (2016). The top 100 high-impact papers in Colombian psychology: A bibliometric study from WoS and Scopus. Informação e Sociedade, 26(2), 137-154. Recuperado de https://www.researchgate. net/publication/309119827_The_top_100_high-impact_papers_in_ Colombian_psychology_A_bibliometric_study_from_WoS_y_Scopus

Díaz, C. (2018). ¿Cómo se construye la noción de ciencia social latinoamericana desde Scopus ? Convergencia entre ensayo y análisis categórico. 28(2), 435-454. DOI: https://doi.org/10.5209/RGID.62833

Escobar-Espinoza, A. y Mercado-Mejía, M. (2014). Las revistas científicas colombianas y la política pública de COLCIENCIAS. Panorama Económico, 22, 7-9. DOI: https://doi.org/10.32997/2463-0470-vol.22num.0-2014-1372 
Fergnani, A. (2019). Mapping futures studies scholarship from 1968 to present: A bibliometric review of thematic clusters, research trends, and research gaps. Futures, 105, 104-123. DOI: https://doi.org/10.1016/j.futures.2018.09.007

Galán, I., Pérez-Gómez, B., \& Primo-Peña, E. (2015). ¿Quién teme al open access? Un movimiento en crecimiento, oportuno y necesario. Gaceta Sanitaria, 29(2), 139-141. DOI: https://doi.org/10.1016/j.gaceta.2014.11.010

Ghadimi, P., Wang, C., \& Lim, M. K. (2019). Sustainable supply chain modeling and analysis: Past debate, present problems and future challenges. Resources, Conservation and Recycling, 140, 72-84. DOI: https://doi. org/10.1016/j.resconrec.2018.09.005

Grupo de Estudios en Diseño Visual. (2004). Presentación. Kepes, 0. Recuperado de http://190.15.17.25/kepes/downloads/Kepes 0.pdf

Hernandez-Garcia, Y. I., Chamizo, J. A., Kleiche-Dray, M., \& Russell, J. M. (2016). The scientific impact of mexican steroid research 1935-1965: A bibliometric and historiographic analysis. Journal of the Association for Information Science and Technology, 67(5), 1245-1256. DOI: https://doi. org/10.1002/asi.23493

Irizar, L. B., Achury, L. E., Pardo, C. N., Camargo, J. G. y Barragán, L. C. (2015). Lo que el dinero no puede comprar... ni la cienciometría medir. Una propuesta humanista del conocimiento frente al mercantilismo cienciométrico. 12, 162-176. DOI: https://doi.org/10.22507/rli.v12n1a15

Keathley-Herring, H., Van Aken, E., Gonzalez-Aleu, F., Deschamps, F., Letens, G., \& Orlandini, P. C. (2016). Assessing the maturity of a research area: bibliometric review and proposed framework. Scientometrics, 109(2), 927-951. DOI: https://doi.org/10.1007/s11192-016-2096-x 
Kepes, G., Giedion, S., \& Hayakawa, S. I. (1967). Language of Vision (B. Books (ed.)). Barakaldo Books. Recuperado de https://monoskop.org/images/3/3b/ Kepes_Gyorgy_El_lenguaje_de_la_vision_1969.pdf

Laengle, S., Modak, N. M., Merigo, J. M., \& Zurita, G. (2018). Twenty-Five Years of Group Decision and Negotiation: A Bibliometric Overview. Group Decision and Negotiation, 27(4), 505-542. DOI: https://doi.org/10.1007/ s10726-018-9582-x

Lawani, S. M. (1981). Bibliometrics: Its Theoretical Foundations, Methods and Applications. Libri, 31(1), 294-315. DOI: https://doi.org/10.1515/ libr.1981.31.1.294

Londoño, F. C. (2005). Presentación. Kepes, 1. Recuperado de http://190.15.17.25/ kepes/downloads/kepes 1.pdf

Modak, N. M., Merigó, J. M., Weber, R., Manzor, F., \& Ortúzar, J. de D. (2019). Fifty years of Transportation Research journals: A bibliometric overview. Transportation Research Part A: Policy and Practice, 120, 188-223. DOI: https://doi.org/10.1016/j.tra.2018.11.015

Moed, H. F. (2009). New developments in the use of citation analysis in research evaluation. Archivum Immunologiae et Therapiae Experimentalis, 57(1), 13-18. DOI: https://doi.org/10.1007/s00005-009-0001-5

Montero, I., \& León, O. G. (2007). A guide for naming research studies in Psychology. International Journal of Clinical and Health Psychology, 7(3), 847-862. Recuperado de https://www.redalyc.org/pdf/337/33770318.pdf

Ordorika, I. (2018). Las trampas de las publicaciones académicas. Revista Española de Pedagogía, 76(271), 463-480. DOI: https://doi.org/10.22550/ REP-3-2018-04 
Ospina, W. (2008). Presentación. Kepes, 4. Recuperado de http://190.15.17.25/ kepes/downloads/kepes4.pdf

Ospina, W. (2010). Presentación. Kepes, 6. Recuperado de http://190.15.17.25/ kepes/downloads/Kepes No. 6.pdf

Paños Álvarez, A. (1999). Reflexiones sobre el papel de la información como recurso competitivo de la empresa. Anales de Documentación: Revista de Biblioteconomía y Documentación, 2, 21-38. DOI: https://doi.org/10.6018/ analesdoc.2.0.2701

Pinski, G., \& Narin, F. (1976). Citation influence for journal aggregates of scientific publications: Theory, with application to the literature of physics. Information Processing and Management, 12(5), 297-312. DOI: https://doi. org/10.1016/0306-4573(76)90048-0

Polanco-Carrasco, R. (2015). Editar para perturbar. Cuadernos de Neuropsicología / Panamerican Journal of Neuropsychology, 9(3), 10-11. Recuperado de http://www.redalyc.org/articulo.oa?id=439643537001\%0D

Pritchard, A. (1969). Statistical bibliography or bibliometrics? Journal of Documentation, 25(4), 348-349. DOI: https://doi.org/10.1108/eb026482

Ramírez-Godoy, M. E., Araiza-Díaz, V. y Díaz-Escoto, A. S. (2019). El Open Access a debate: entre el pago por publicary la apertura radical sostenible. 33, 195-216.DOI:http://dx.doi.org/10.22201/iibi.24488321xe.2019.80.58039

Ray Reutzel, D., \& Mohr, K. A. J. (2015). 50 years of reading research quarterly (1965-2014): Looking back, moving forward. Reading Research Quarterly, 50(1), 13-35. DOI: https://doi.org/10.1002/rrq.87 
Rodríguez Piña, R. A. (2006). Metodología para el análisis de información orientada al análisis de tendencias en el Web superficial a partir de fuentes no estructuradas.: Parte I. Fundamentos teóricos. Acimed, 14(6), 0. Recuperado de http://scielo.sld.cu/scielo.php?pid=S102494352006000600005\&script=sci_arttext

Salas, G., Ponce, F. P., Méndez-Bustos, P., Vega-Arce, M., Pérez, M. de los Á., López-López, W. y Cárcamo-Vásquez, H. (2017). 25 Años de Psykhe: Un Análisis Bibliométrico. Psykhe, 26(1), 1-17. DOI: https://doi.org/10.7764/ psykhe.26.1.1205

Santos, V. E. (2010). Difusión y divulgación de la investigación científica. Idesia, 28(3), 5-6. https://doi.org/http://dx.doi.org/10.4067/S071834292010000300001

Scimago Journal \& Country Rank. (2019). KEPES. Recuperado de https://www.scimagojr.com/journalsearch.php?q=21100244946\&tip=sid\& clean $=0$

Serrano, M. O. (2016). La etapa inicial de la Revista Mexicana de Ciencias Políticas y Sociales a 60 años de distancia. Prácticas conmemorativas, legados, olvidos y nuevos pasados/presentes. Revista Mexicana de Ciencias Politicas y Sociales, 61(226), 427-456. DOI: https://doi.org/10.1016/S01851918(16)30017-4

Stelzer, B., Meyer-Brötz, F., Schiebel, E., \& Brecht, L. (2015). Combining the scenario technique with bibliometrics for technology foresight: The case of personalized medicine. Technological Forecasting and Social Change, 98, 137-156. DOI: https://doi.org/10.1016/j.techfore.2015.06.008

Subramanyam, K. (1983). Bibliometric studies of research collaboration_A review_Subramanyam.pdf. Journal of Information Science, 6(1), 33-38. DOI: http://journals.sagepub.com/doi/abs/10.1177/016555158300600105 
Tur-Porcar, A., Mas-Tur, A., Merigó, J. M., Roig-Tierno, N., \& Watt, J. (2018). A Bibliometric History of the Journal of Psychology Between 1936 and 2015. The Journal of Psychology, 152(4), 199-225. DOI: https://doi.org/10 .1080/00223980.2018.1440516

Uribe-Bahamonde, Y., \& Díaz Herrera, C. (2019). UNIVERSUM after 32 years of its foundation: Bibliometric and relational analysis. Universum (Talca), 34(1), 217-245. DOI: https://doi.org/10.4067/S0718-23762019000100217

Uribe-Tirado, A. y Alhuay-Quispe, J. (2017). Estudio métrico de ALFIN en Iberoamérica: de la bibliometría a las altmetrics. Revista Española de Documentación Científica, 40(3), 180. DOI: https://doi.org/10.3989/ redc.2017.3.1414

Valderrama, J. O. M. (2012). Aspectos éticos en las publicaciones de revistas científicas de corriente principal. Revista Chilena de Pediatria, 83(5), 417-419. DOI: https://doi.org/10.4067/S0370-41062012000500001

Van Raan, A. F. J. (1997). Scientometrics: State-of-the-art. Scientometrics, 38 (1), 205-218. DOl: https://doi.org/10.1007/BF02461131

Villa Enciso, E. M., Valencia Arias, J. y Valencia Arias, A. (2016). El papel de las narrativas digitales como nueva estrategia educativa: resultados desde un análisis bibliométrico. KEPES, 13(13), 197-231. DOI: https://doi. org/10.17151/kepes.2016.13.13.10

Villa, G. (2011). Presentación. KEPES, 7(8), 5-6 Recuperado de http://190.15.17.25/kepes/downloads/Revista7_Completo.pdf

Villa, G. (2013). Presentación. KEPES, 8(9), 5-6 Recuperado de http://190.15.17.25/kepes/downloads/Revista9_Completa.pdf

Villa, G. (2014). Presentación. KEPES, 9(10), 5-8 Recuperado de http://190.15.17.25/kepes/downloads/Revista10_Completo.pdf 
Villa, G. (2015a). Presentación. KEPES, 10(11), 5-8. DOI: 10.17151/ kepes.2015.12.11.1

Villa, G. (2015b). Presentación. KEPES, 12(12), 5-10. DOI: 10.17151/ kepes.2015.12.12.1

Vinkler, P. (1993). Research contribution, authorship and team cooperativeness. Scientometrics, 26(1), 213-230. DOI: https://doi.org/10.1007/BF02016801

Wren, J. D. (2005). Open access and openly accessible: a study of scientific publications shared via the internet. Bmj, 330(7500), 1128-0. DOI: https://doi.org/10.1136/bmj.38422.611736.E0

Yeong, C. H. \& Abdullah, B. J. J. (2012). Altmetrics: The right step forward. Biomedical Imaging and Intervention Journal, 8(2), 1-2. DOI: https://doi. org/10.2349/biij.8.3.e15

Como citar: Uribe, Y. (2020). KEPES, desde Colombia al mundo. Una tarea pendiente: análisis bibliométrico y relacional. Revista KEPES, 17(22), 17-58. https://doi.org/10.17151/kepes.2020.17.22.2 